\title{
Observing the development of the temporomandibular joint in embryonic and post-natal mice using various staining methods
}

\author{
WENNA LIANG $^{1 *}$, XIHAI LI $^{2^{*}}$, BIZHEN GAO $^{1}$, HUIJUAN GAN $^{1}$, \\ XUEJUAN LIN ${ }^{1}$, LINGHONG LIAO ${ }^{1}$ and CANDONG LI ${ }^{1}$ \\ ${ }^{1}$ College of Traditional Chinese Medicine, Research Base of Traditional Chinese Medicine Syndrome; \\ ${ }^{2}$ Academy of Integrative Medicine, Institute of Bone Diseases, Fujian University of Traditional Chinese Medicine, \\ Fuzhou, Fujian 350122, P.R. China
}

Received July 16, 2014; Accepted November 25, 2015

DOI: $10.3892 / \mathrm{etm} .2015 .2937$

\begin{abstract}
The temporomandibular joint (TMJ) is a specialized synovial joint that is essential for the movement and function of the mammalian jaw. The TMJ develops from two mesenchymal condensations, and is composed of the glenoid fossa that originates from the otic capsule by intramembranous ossification, the mandibular condyle of the temporal bone and a fibrocartilagenous articular disc derived from a secondary cartilaginous joint by endochondral ossification. However, the development of the TMJ remains unclear. In the present study, the formation and development of the mouse TMJ was investigated between embryonic day 13.5 and post-natal day 180 in order to elucidate the morphological and molecular alterations that occur during this period. TMJ formation appeared to proceed in three stages: Initiation or blastema stage; growth and cavitation stage; and the maturation or completion stage. In order to investigate the activity of certain transcription factors on TMJ formation and development, the expression of extracellular matrix (ECM), sex determining region Y-box 9 , runt-related transcription factor 2, Indian hedgehog homolog, Osterix, collagen I, collagen II, aggrecan, total matrix metalloproteinase (MMP), MMP-9 and MMP-13 were detected in the TMJ using in situ and/or immunohistochemistry. The results indicate that the transcription factors, ECM and MMP serve critical functions in the formation and development of the mouse TMJ. In summary, the development of the mouse TMJ was investigated, and the molecular regulation of mouse TMJ formation was partially characterized. The results of
\end{abstract}

Correspondence to: Dr Candong Li, College of Traditional Chinese Medicine, Research Base of Traditional Chinese Medicine Syndrome, Fujian University of Traditional Chinese Medicine, 1 Qiuyang Road, Fuzhou, Fujian 350122, P.R. China

E-mail: fjzylcd@126.com

* Contributed equally

Key words: temporomandibular joint, glenoid fossa, condyle, articular disc, development the present study may aid the systematic understanding of the physiological processes underlying TMJ formation and development in mice.

\section{Introduction}

The temporomandibular joint (TMJ) is a complex skeletal structure that is essential for jaw movement in mammals (1). The TMJ is comprised of multiple tissues, including the mandibular condyle, glenoid fossa, a fibrocartilaginous articular disc located between these two bones that divides the joint cavity into two compartments, and a variety of associated tendons and muscles $(2,3)$. Furthermore, the tendons of the pterygoid muscle and various surrounding ligaments are associated with the TMJ (4). Disorders of the TMJ affect numerous individuals, and may lead to difficulty in chewing function and chronic myofacial pain (5).

The embryonic development of the TMJ shares a similar development process across various mammalian species, and differs significantly from that of other synovial joints (6). In contrast with the formation of long bone joints by cleavage or segmentation within a single skeletal condensation, the TMJ develops from two distinct and widely separated mesenchymal condensations; the glenoid fossa blastema and the condylar blastema (7). The glenoid fossa blastema derives from the otic capsule and undergoes transmembranous ossification $(8,9)$. The condylar blastema develops rapidly towards a rectangular cell condensation located lateral to and above Meckel's cartilage, and is subsequently attached medially by the lateral pterygoid muscle as a result of rapid cellular proliferation (4). Simultaneously, the condylar blastema develops out of the secondary condyle cartilage of the mandible and forms a bone via endochondral ossification, subsequently extending in an anterior/medial direction and capping the condylar blastema $(10,11)$.

The intervening mesenchyme between the glenoid fossa and condylar blastemas condenses, prior to the separation of the two primordia of the TMJ by an articular disc (12). As the condyle develops continuously upward approaching the glenoid fossa, the mesenchyme differentiates into layers of fibrous tissues, ultimately separating the upper and lower synovial cavities (13). In addition to cellular proliferation 
and differentiation, the condyle anlage is configured into a typical secondary cartilage and is superficially covered with a thick layer of flat fibrous cells $(14,15)$. The glenoid fossa exhibits intramembranous ossification, which corresponds to condyle differentiation (8). During the development of the skeletal elements of the TMJ, morphogenesis of the soft tissues surrounding the joint continues (16). Following the completion of cavitation, the TMJ exhibits marked ossification and growth of the condyle and glenoid fossa, functional remodeling of the articular disc via an avascular event and substantial condensation (17). Furthermore, enclosure of the joint bone prominences and the articular disc through the joint capsule occurs, and the development of the muscles and ligaments proceeds $(18,19)$. Although the structure and function of the TMJ has been well characterized (12-19), the molecular and cellular mechanisms underlying its formation and development remain unclear. Therefore, the aim of the present study clarify was to investigate the processes underlying the formation and development of the mouse TMJ using various immunohistochemical staining methods.

\section{Materials and methods}

Animal preparation. A total of 30 pregnant BALB/c mice, aged 2 months and weighing 33.46 $\pm 3.57 \mathrm{~g}$, were purchased from the Shanghai Slack Laboratory Animal Co., Ltd. (Shanghai, China). Mice were housed under a 12-h light-dark cycle at $22 \pm 1^{\circ} \mathrm{C}$ and with a humidity of $56 \pm 5 \%$, with 5 mice per cage, and received a $0.3 \%$ sodium diet, ad libitum. Animal experiments were conducted in accordance with the Guide for Care and Use of Laboratory Animals of Fujian University of Traditional Chinese Medicine (Fuzhou, China). The age of the mouse embryo was defined based on the ejection of the vaginal plug from the mother. The day of the morning following this occurrence was defined as embryonic day 0.5 (E0.5). Pregnant, embryonic and post-natal mice were sacrificed using carbon dioxide (cage size, $7 \times 11 \times 5$ inches; flow rate, 1.31/min), according to the Guide for the Care and Use of Animals (8th edition, 2011). Embryos were subsequently extracted in phosphate-buffered saline (PBS; pH 7.4; GE Healthcare Life Sciences, Logan, UT, USA). Following collection, embryonic mice heads were stored in ice-cold phosphate-buffered saline. Heads collected on days E13.5, E14.5 and E15.5 were fixed in $4 \%$ paraformaldehyde (Sigma-Aldrich, St. Louis, MO, USA) and PBS overnight at $4^{\circ} \mathrm{C}$, while heads collected on days E16.5, E17.5, E18.5 and post-natal days 0 (P0), P7, P14, P21 and P180 were fixed and decalcified using a Surgipath Decalcifier I (Leica Microsystems GmbH, Wetzlar, Germany) for various periods of time depending on the age of the mouse (2).

Histological analyses. Mouse heads were dehydrated using a graded ethanol series, cleared with xylene, embedded in paraffin (both Sigma-Aldrich) and sectioned at $10 \mu \mathrm{m}$ using a Leica RM2235 microtome (Leica Microsystems GmbH). For histological analysis of the TMJ, serial sections were subjected to standard hematoxylin-eosin (HE) (20), Safranin O-Fast green (21) and Azon red/Anilin blue staining (2,9) (all Sigma-Aldrich), according to previously described methods. HE staining is essential for recognizing various tissue types and morphological alterations, and it facilitates visualiza- tion of a range of nuclear, cytoplasmic and extracellular matrix (ECM) features. Hematoxylin stains nucleic acids a deep blue-purple color, whereas eosin is pink and stains proteins non-specifically. Therefore, in a typical tissue, nuclei are stained blue and the cytoplasm and ECM components exhibit varying degrees of pink staining. Safranin O/Fast green staining is frequently used to stain articular cartilage. Safranin $\mathrm{O}$ is a cationic dye that stains acidic proteoglycans present in cartilage tissues, which are indicators of cell chondrogenesis, with an orange-red color. Fast green, which is the contrast stain of Safranin $\mathrm{O}$, is a sulfate group containing acidic substrate that binds to protein amino groups, thereby staining non-collagen sites. Azon red/Anilin blue is a double stain containing Alizarin (bone) and alcian blue (cartilage) compounds which is used to identify cartilage and bone.

In situ hybridization and immunohistochemistry. Mouse heads were embedded in paraffin and sectioned at $10 \mu \mathrm{m}$ for in situ and $8 \mu \mathrm{m}$ for immunohistochemical analysis. Non-radioactive riboprobes, including SOX-9 [nucleotides (nt), 116-856; NM_011448), RUNX2 (nt, 3183-3812; NM_001146038), Osterix (nt, 40-1727; NM_130458) and IHH (nt, 897-1954; NM_010544), were synthesized using in vitro transcription labeling with Digoxigenin-11-UTP, according to the manufacturer's instructions (Roche Diagnostics GmbH, Mannheim, Germany) (9). Briefly, $10 \mu \mathrm{m}$ sections were pretreated with $10 \mu \mathrm{g} / \mathrm{ml}$ proteinase $\mathrm{K}$ (Sigma-Aldrich), fixed in $4 \%$ paraformaldehyde, hybridized with riboprobes at $50^{\circ} \mathrm{C}$ for $16 \mathrm{~h}$, and washed with $2 \mathrm{X}$ standard saline citrate (Sigma-Aldrich) containing $50 \%$ formamide (Sigma-Aldrich) at $50^{\circ} \mathrm{C}$. Maleic acid buffer and blocking reagent (Roche Diagnostics $\mathrm{GmbH}$ ) were added for blocking and antibody washing steps. Signals were developed with BM purple alkaline phosphatase substrate (Sigma-Aldrich). Immunohistochemical staining was performed according to the manufacturer's instructions (2). Paraffin sections were deparaffinized and rehydrated in a descending series of alcohol dilutions, heated in $10 \mathrm{mM}$ sodium citrate buffer ( $\mathrm{pH}$ 6.0; Sigma-Aldrich) at $100^{\circ} \mathrm{C}$ for $20 \mathrm{~min}$ for antigen retrieval, then cooled to room temperature. The sections were blocked with goat serum (1:10; Invitrogen; Thermo Fisher Scientific, Inc., Carlsbad, CA, USA), and incubated for $15 \mathrm{~min}$ at room temperature. Subsequently, the sections were incubated with polyclonal antibodies against runt-related transcription factor 2 (RUNX2; 1:1,000; ab76956), sex determining region Y-box 9 (SOX-9; 1:500; ab26414), collagen I (1:500; ab34710), collagen II (1:200; ab53047), aggrecan (1:500; ab36861), matrix metalloproteinase-9 (MMP-9; 1:300; ab38898), MMP-13 (1:50; ab75606) and Indian hedgehog homolog (IHH; 1:200; ab39634) from Abcam (Cambridge, MA, USA) overnight at $4^{\circ} \mathrm{C}$. The slides were then washed three times using PBS; and incubated with a biotinylated horseradish peroxidase goat anti-rabbit secondary antibody (1:1,000; A-11034; Invitrogen; Thermo Fisher Scientific, Inc.) for $20 \mathrm{~min}$ at $37^{\circ} \mathrm{C}$. The slides were washed three times following incubation with the secondary antibody using PBS. Immunolabeling was visualized with $0.05 \%$ diaminobenzidine (Invitrogen; Thermo Fisher Scientific, Inc.) in PBS for $5 \mathrm{~min}$ at room temperature, then slides were rinsed for $10 \mathrm{~min}$ under running tap water. The morphology of immunohistochemically stained TMJ sections 

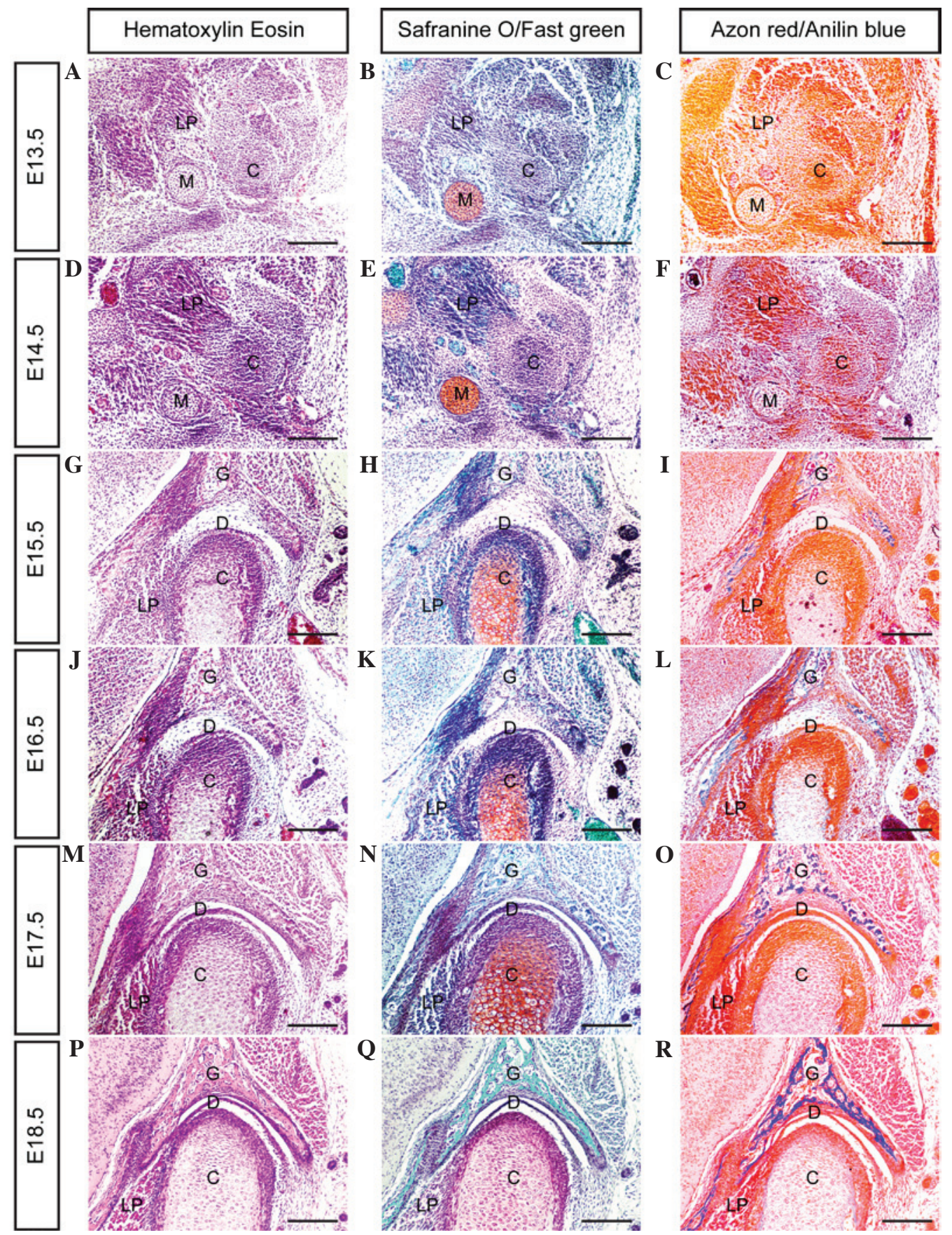

Figure 1. Histological analysis showing the temporomandibular joint (TMJ) formation during the embryonic stage in mice. (A-R) Coronal sections through the TMJ show the process of TMJ formation between days E13.5 and E18.5 (scale bar, $100 \mu \mathrm{m}$ ). E, embryonic day; G, glenoid fossa; C, condyle; D, disc; LP, lateral pterygoid muscle; M, Meckel's cartilage.

was observed using a BH-2 light microscope (Olympus Corporation, Tokyo, Japan).

In situ zymography and 4'6'-diamidino-2-phenylindole dihydrochloride (DAPI) staining. Heads of the P0 mice were immersed in zinc-based fixative containing $36.7 \mathrm{mM}$ $\mathrm{ZnCl}, 27.3 \mathrm{mM} \mathrm{ZnAc} \cdot 2 \mathrm{H}_{2} \mathrm{O}$ and $0.63 \mathrm{mM}$ calcium acetate in $0.1 \mathrm{mM}$ Tris (pH 7.4; Sigma-Aldrich) for $2 \mathrm{~h}$ at room temperature, dehydrated using 15 and $30 \%$ sucrose at $4^{\circ} \mathrm{C}$ overnight, frozen in optimal cutting temperature compound (Sakura Finetek USA, Inc., Torrance, CA, USA), then sectioned at $10 \mu \mathrm{m}$ using a Leica CM1850 cryostat (Leica Microsystems
GmBH). DQ-gelatin (1 mg/ml; E12055; Molecular Probes; Thermo Fisher Scientific, Inc., Grand Island, NY, USA) was used as the substrate at 1:10 dilution in the in situ zymography buffer, according to the manufacturer's instructions (2). Next, $100 \mu \mathrm{l}$ mixture was applied to the sections, which were incubated at $37^{\circ} \mathrm{C}$ for $2 \mathrm{~h}$ in a dark humid chamber. In order to visualize the DNA in the frozen sections, sections were incubated with $100 \mathrm{ng} / \mathrm{ml}$ DAPI (Sigma-Aldrich) in PBS for $30 \mathrm{~min}$. The gelatinolytic activity and DAPI-stained TMJ frozen sections were observed as green fluorescence using a Axioskop 50 fluorescence microscope (Carl Zeiss AG, Oberkochen, Germany). 


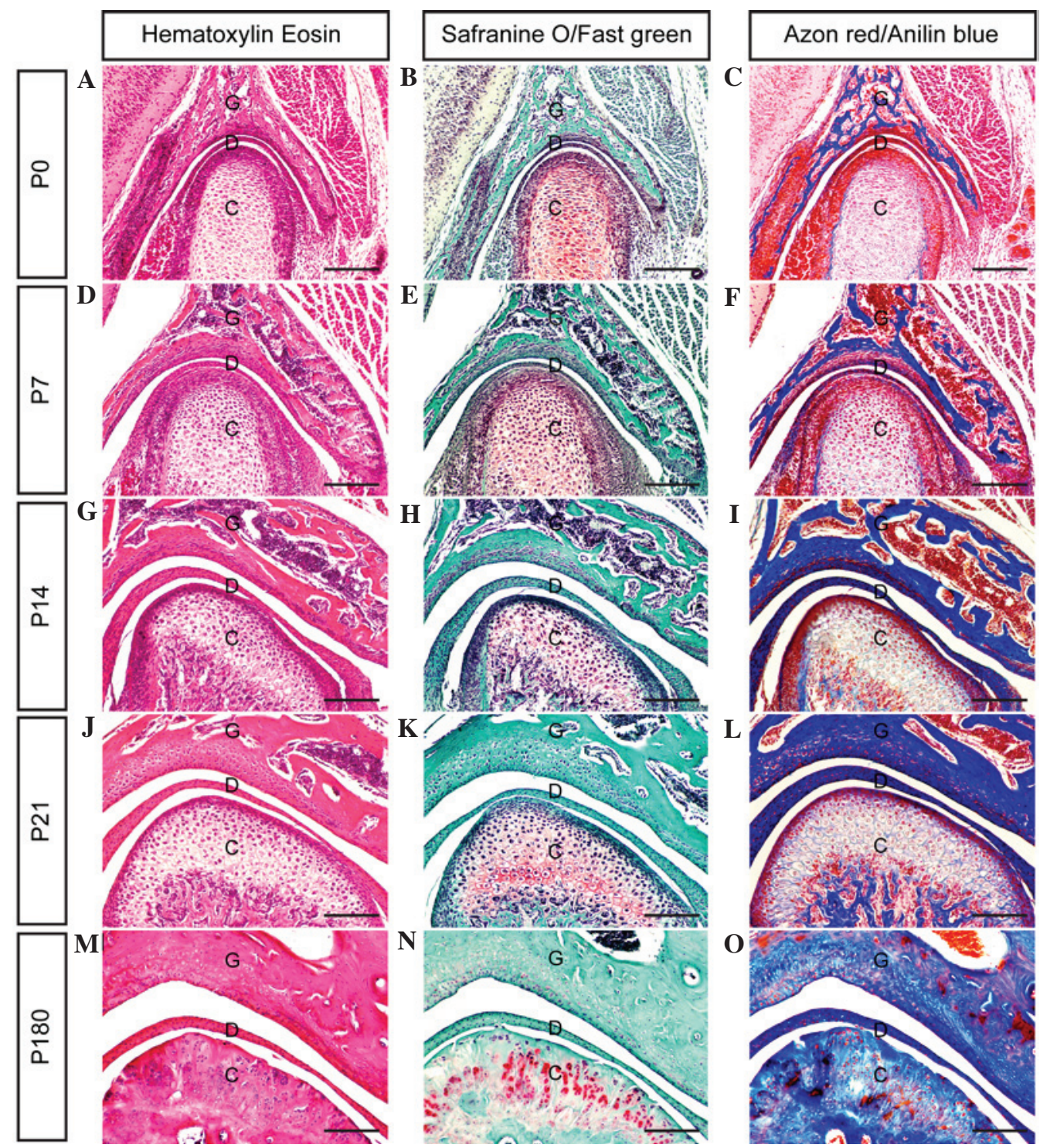

Figure 2. Histological analyses showing the development of the temporomandibular joint (TMJ) during the post-natal stage in mice. (A-O) Coronal sections through the TMJ show the process of TMJ development between days P0 and P180 (scale bar, $100 \mu \mathrm{m}$ ). G, glenoid fossa; C, condyle; D, disc; P, post-natal day 8 .

Bromodeoxyuridine (BrdU) labeling for cell proliferation, and terminal deoxynucleotidyl transferase dUTP nick-end labeling (TUNEL) for cell apoptosis assays. Six pregnant mice were injected with labeling reagent $(1.5 \mathrm{ml} / 100 \mathrm{~g})$ from a BrdU Labeling Detection Kit II (Roche Diagnostics $\mathrm{GmbH}$ ) for $2 \mathrm{~h}$ prior to sacrifice using carbon dioxide euthanasia. Subsequently, three heads of E13.5-E18.5 were fixed in Carnoy's fixative containing ethanol absolute (Merck \& Co., Inc., Whitehouse Station, NJ, USA), chloroform (Sigma-Aldrich) and glacial acetic acid (Merck \& Co., Inc.) at a ratio of $6: 3: 1$. The heads were then ethanol-dehydrated, paraffin-embedded and sectioned at $5 \mu \mathrm{m}$. Sections were subjected to immunodetection for analysis of cellular proliferation, according to the manufacturer's instructions (9). Heads of E13.5-E18.5 were subjected for cell proliferation assay, and three adjacent sections from each head were observed for BrdU labeling. Three heads of the P0 mice were sectioned at $5 \mu \mathrm{m}$, and subjected to immunodetection for cell apoptosis analysis, according to the manufacturer's instructions (2). Apoptosis was measured using an in situ
Cell Death Detection Kit (Roche Diagnostics GmbH), and three adjacent sections from each head were observed using TUNEL labeling.

Statistical analysis. Statistical analyses were performed using the Student's t-test with SPSS software, version 13.0 (SPSS, Inc., Chicago, IL, USA). Data were expressed as the mean \pm standard deviation. $\mathrm{P}<0.05$ was considered to indicate a statistically significant difference.

\section{Results and Discussion}

Formation of the TMJ during the embryonic development in mice. In mice, the mesenchymal condensation of the condyle develops at day E13.5 (Fig. 1A-C); however, the glenoid fossa primordium does not form by this time point. The location of the glenoid fossa is indicated by the surrounding lateral pterygoid muscle, trigeminal ganglion and Meckel's cartilage. In serial coronal sections, the posterior portion of the condylar primordium appears to be isolated from the mandible, while 


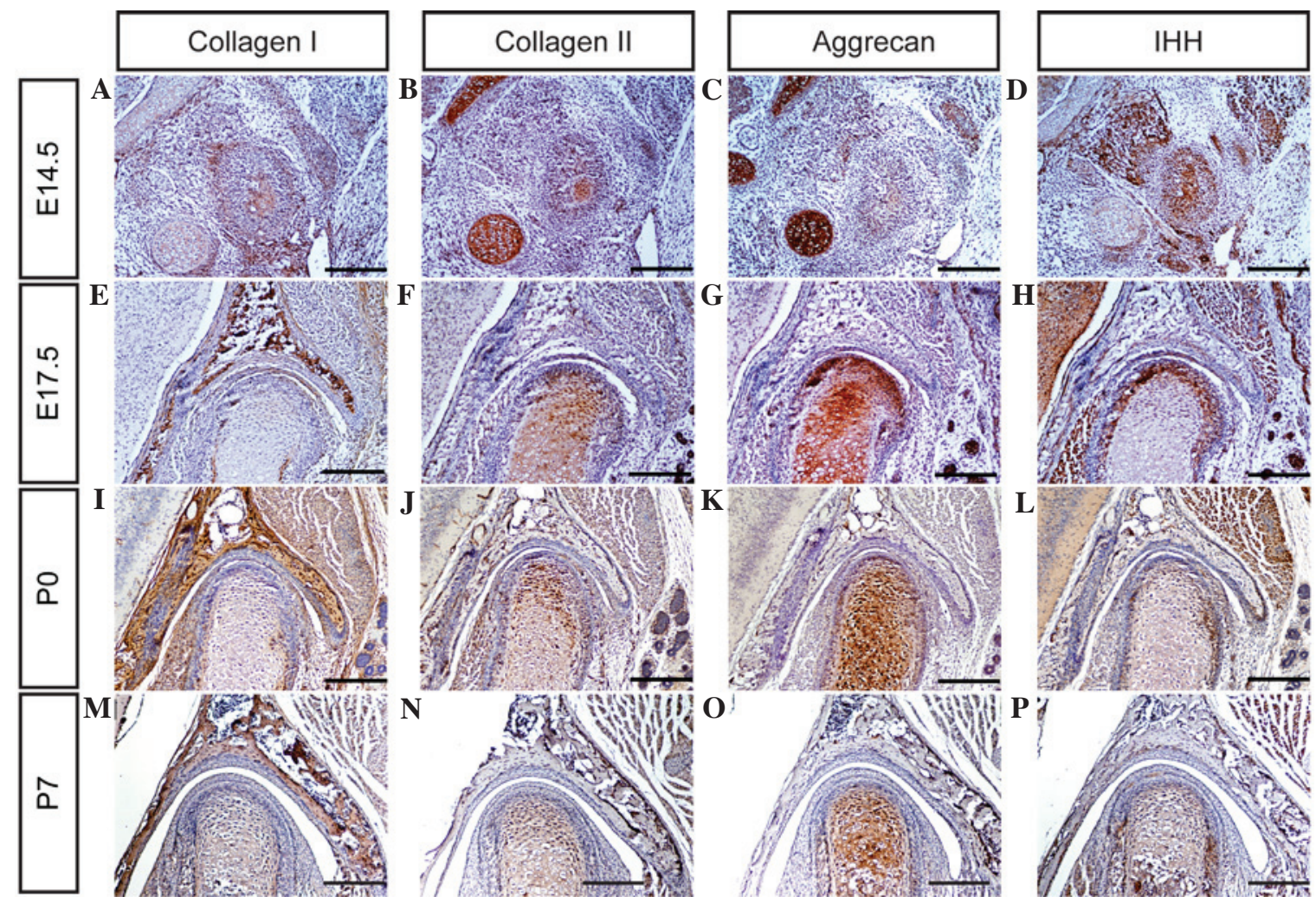

Figure 3. Collagen I, collagen II, aggrecan and IHH expression was detected in the temporomandibular joint using immunohistochemistry at (A-D) E14.5, (E-H) E17.5, (I-L) P0 and (M-P) P7 (scale bar, $100 \mu \mathrm{m})$. IHH, Indian hedgehog homolog; E, embryonic day; P, post-natal day.

its anterior portion is closely connected to the primordium of the mandible.

By day E14.5 (Fig. 1D-F), the condylar primordium is rearranged and shaped into a reverse positioned cone, with a tip pointed toward the mandibular bone. Medially, the lateral pterygoid muscle runs into the cone-shaped condyle. Above the condyle, the zygomatic process and squamosal plate combine to form a concave-shaped glenoid fossa primordium.

By day E15.5 (Fig. 1G-I), the cartilage that has developed in the center of the condyle is surrounded by a thick layer of perichondrium. At the apex of condyle development, numerous layers of cells from the surface of the condyle are compacted into a strip, representing the articular disc anlage. Furthermore, the glenoid fossa becomes enlarged, and begins to ossify in the center of the fossa. However, the space between the developing condyle and glenoid fossa remains wide.

At day E16.5 (Fig. 1J-L) a cleft, generated by the upward movement of loose mesenchymal cells in the articular space, forms between the glenoid fossa and articular disc, indicating the initiation of cavitation. At this point there is no indication of the formation of lower cavitation; however, the strip of disc anlage is separated completely from the condyle surface. Furthermore, the cells of the medial area of the disc anlage combine with the lateral pterygoid muscle cells. The rapidly developing condyle differentiates to form various layers, including fibrous, polymorphic, flattened and hypertrophic cell layers. The rapid growth of the condyle and glenoid fossa leads to the narrowing of the articular space.

At day E17.5 (Fig. 1M-O), the formation of a definite articular disc with numerous tightened layers of fiber results in the formation of a narrow cavity between the condyle and articular disc. The lateral fiber tangle of the articular disc, with the tendon fibers derived from the masseter muscle, and its medial fibers combine with the tendon fibers extended from the lateral pterygoid muscle.

At day E18.5 (Fig. 1P-R), all of the major anatomical features of the TMJ are present. A definite and compact articular disc is clearly present, separating the upper and lower synovial cavities. As shown in Fig. 2, the present results clarify the stages of TMJ development from immaturity to maturity in post-natal mice.

Regulation of TMJ development by ECM. The ECM appears to serve crucial functions in TMJ development, in addition to those served by transcription and growth factors. TMJ structure-function associations are described in terms of its most abundant ECM components, including collagen, glycosaminoglycans and proteoglycans $(22,23)$. These ECM components are critical for resistance against compressive forces and for maintaining the tensile properties of the tissue, and are increased or decreased in a number of cartilage pathologies and across the different stages of a single pathology (24).

Collagen belongs to a family of structural proteins with distinct chemical compositions, morphologies and functions (25). To date, $>27$ types of collagen have been classified (25). Certain types of collagen, including collagen I, II and IV, are capable of aggregating molecules to form the articular disc, condyle and glenoid fossa of the TMJ $(26,27)$. Aggrecan, the best understood of the lecticans, is fundamental for cartilage function and skeletal development $(28,29)$. The biological function of aggrecan is the ability to bind hyaluronan in order to provide the basis for the viscoelastic 


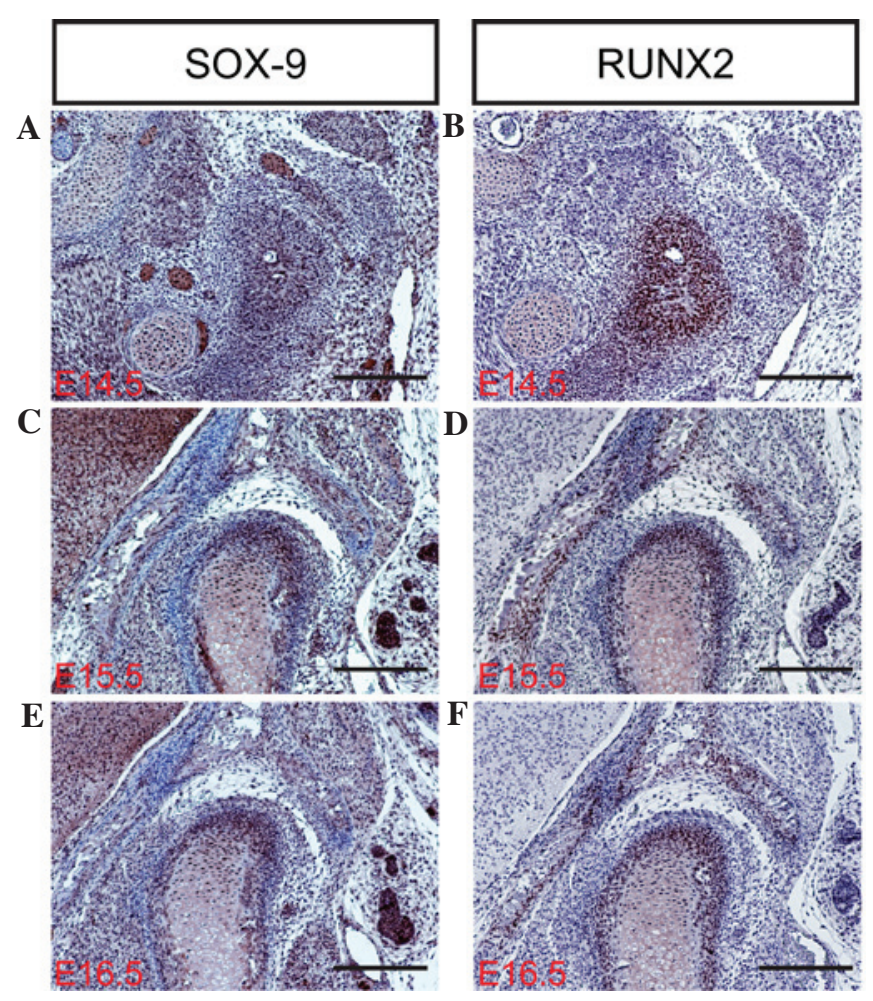

Figure 4. SOX-9 and RUNX2 expression detected during temporomandibular joint development using immunohistochemistry at (A and B) E14.5, (C and D) E15.5 and (E and F) E16.5 (scale bar, $100 \mu \mathrm{m}$ ). SOX-9, sex determining region Y-box 9; RUNX2, runt-related transcription factor 2; E, embryonic day.

properties necessary for load distribution over the articular surface (28). In addition, aggrecans are crucially involved in skeletal development, as a key molecular component of the cartilage templates in the process of endochondral ossification (29). In order to investigate the alternation of ECM during TMJ development in the present study, protein expression levels of collagen I, collagen II and aggrecan in the TMJ were determined using immunohistochemistry. It was observed that collagen I, collagen II and aggrecan are expressed weakly in the TMJ at day E14.5 (Fig. 3A-C), and markedly by days E17.5, P0 and P7 (Fig. 3E-G, I-K and M-O, respectively). These observations suggest that all the major ECM components of the TMJ are well-formed by these time points, and may therefore serve critical functions in TMJ development in mice.

Regulation of TMJ development by transcription factors. During TMJ development, a large number of transcription factors and growth factors have been implicated in the development of primary cartilage and endochondral ossification, including SOX-9, RUNX2, IHH and Osterix (30-32). The transcription factor SOX-9 is expressed in mesenchymal condensations and proliferating chondrocytes of the condyle. Targeted inactivation of SOX-9 in neural crest cells results in the complete ablation of the condyle due to the failed formation of the condylar blastema (30-33). In contrast with SOX-9, the RUNX2 transcription factor appears to serve a distinct function in the development of the condylar and primary cartilage. RUNX2 is initially expressed in the mesenchymal condensation of the condyle, and subsequently in the newly formed cartilage and bone collar, which is similar to its
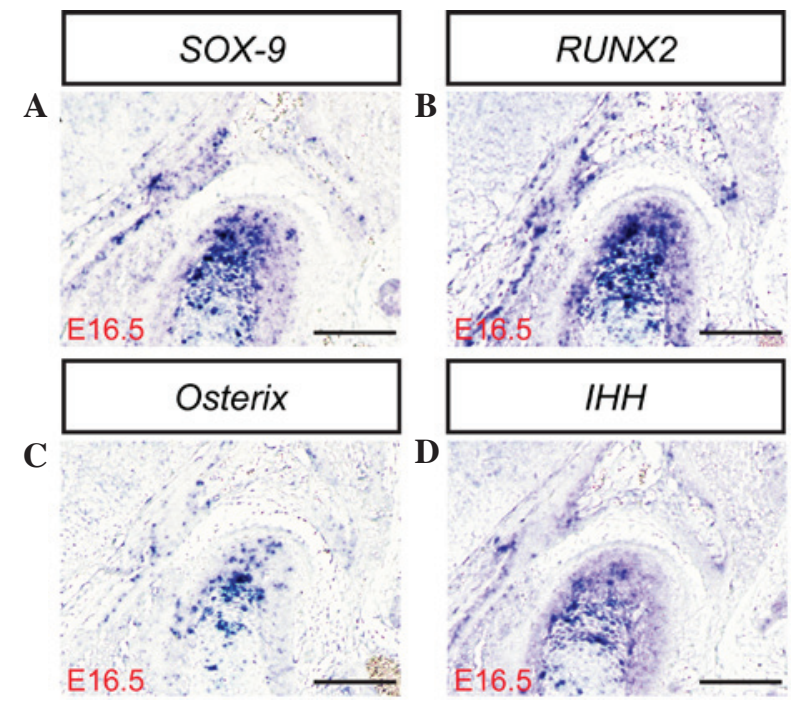

Figure 5. (A) SOX-9, (B) RUNX2, (C) Osterix and (D) IHH expression measured in the temporomandibular joint at E16.5 in situ (scale bar, $100 \mu \mathrm{m}$ ). SOX-9, sex determining region Y-box 9; RUNX2, runt-related transcription factor 2; IHH, Indian hedgehog homolog; E, embryonic day.

expression during long bone formation (9). Osterix, a novel zinc-finger-containing transcription factor, is another essential factor for osteoblast differentiation and functions downstream of RUNX2 in the lineage of osteoblast differentiation. RUNX2 and Osterix are essential for primary cartilage or bone formation. RUNX2 is primarily involved in the differentiation of mesenchymal cells into preosteoblasts (34), whereas Osterix is associated with the differentiation of preosteoblasts into mature osteoblasts (30). IHH, a member of the Hedgehog family, serves a pivotal role in long bone development and digit joint formation (24). IHH regulates chondrocyte proliferation and the rate of chondrocyte hypertrophy in cooperation with parathyroid hormone-related protein (PTHrP) in the periarticular region $(1,35)$. In mice, IHH expression is initially detected in the condylar condensation, and becomes marked in the condylar cartilage, in addition to PTHrP expression, at day E15.5 $(36,37)$, suggesting that the IHH-PTHrP regulatory loop operates in the developing condyle. In the present study, the mRNA and protein expression levels of SOX-9, RUNX2, IHH and Osterix (Fig. 3Q) were determined in the mouse TMJ using in situ hybridization and/or immunohistochemistry (Figs. 3D, H, L and P; 4A-F; and 5A-D). The results of the present study indicate that SOX-9, RUNX2, IHH and Osterix are expressed in the TMJ at different stages of development. These results are consistent with those of previous studies $(2,6,7,9)$, which indicate that transcription factors are important regulators of the formation of the TMJ during the various stages of mouse embryonic development.

Regulation of TMJ development by cell proliferation and apoptosis. Cells proliferate throughout ontogenesis to facilitate tissue remodeling and renewal, and the repair of damaged areas during wound healing (6). Cell proliferation involves replication by normal complete division cycles, which are sequential and associated with the cell cycle $(7,9)$. Cell proliferation may be evaluated using a labeling index (such as the percentage of cells in $\mathrm{S}$ phase) following the administration 

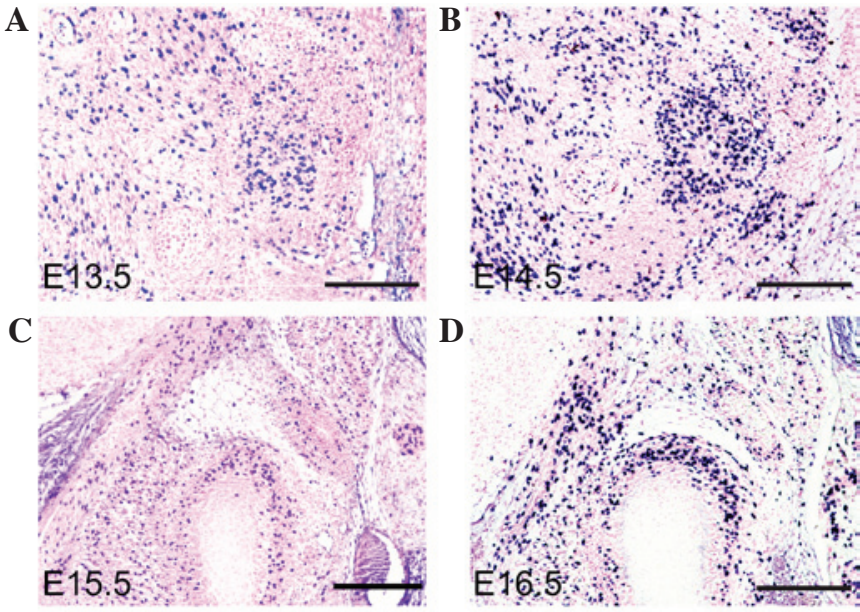

$\mathbf{E}$
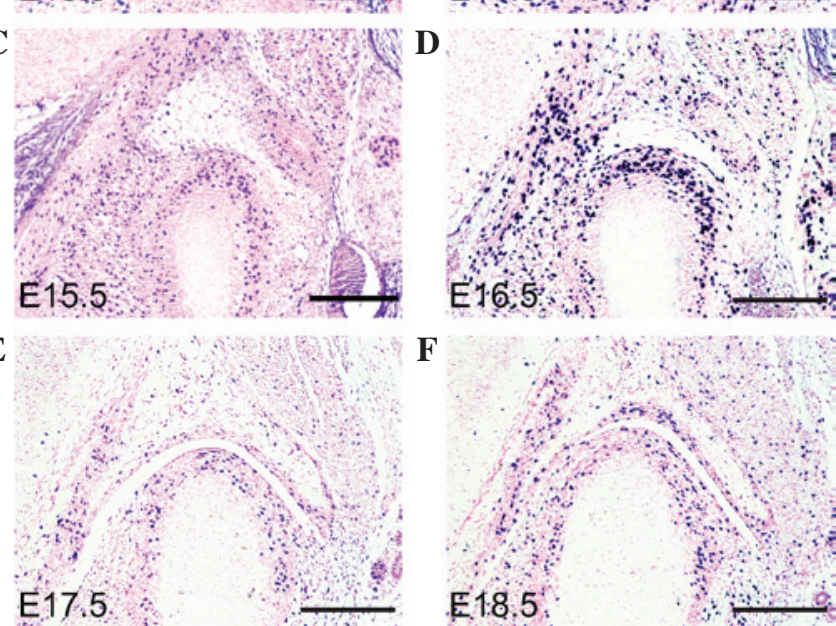

Figure 6. Differences in cell proliferation in the temporomandibular joint (TMJ) during development. Differences were evaluated using a bromodeoxyuridine assay at (A) E13.5, (B) E14.5, (C) E15.5, (D) E16.5, (E) E17.5 and (F) E18.5 (scale bar, $100 \mu \mathrm{m}$ ). E, embryonic day.
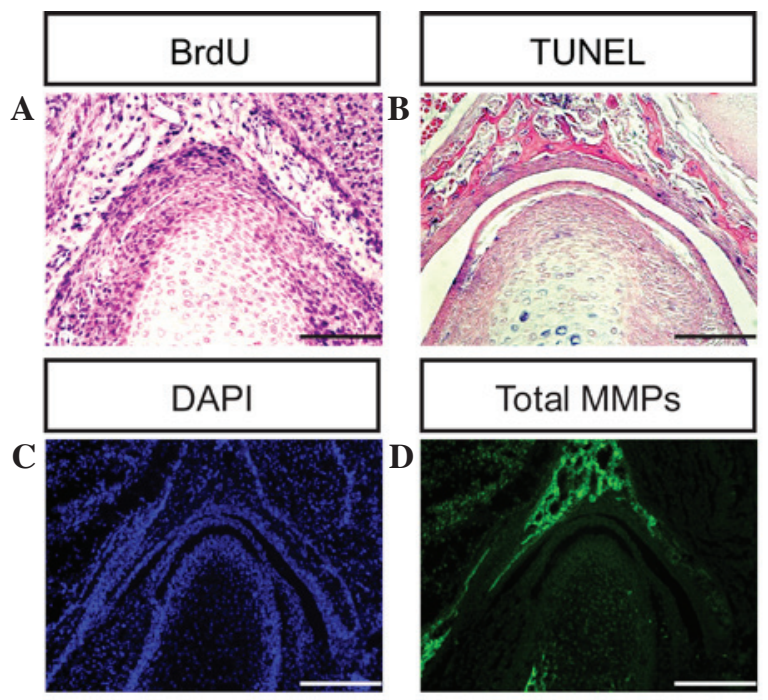

Figure 7. Differences in cell proliferation, apoptosis and total MMPs during temporomandibular joint (TMJ) development. Differences were evaluated using (A) BrdU, (B) TUNEL and (C) DAPI assays at post-natal day 0. (D) Total MMP expression was measured at P0 using in situ zymography (scale bar, $100 \mu \mathrm{m})$. BrdU, bromodeoxyuridine; TUNEL, terminal deoxynucleotidyl transferase dUTP nick end labeling; DAPI, 4',6-diamidino-2-phenylindole; MMP, matrix metalloproteinase.

of DNA precursor labels such as tritiated thymidine or BrdU, or by immunostaining the endogenous cell replication marker proliferating cell nuclear antigen $(38,39)$. In order to investigate differences in cellular proliferation during TMJ development, a BrdU assay was used to evaluate cellular proliferation in the condyle and glenoid fossa of the TMJ in the present study (Figs. 6A-F and 7A). The cells of the condyle and glenoid

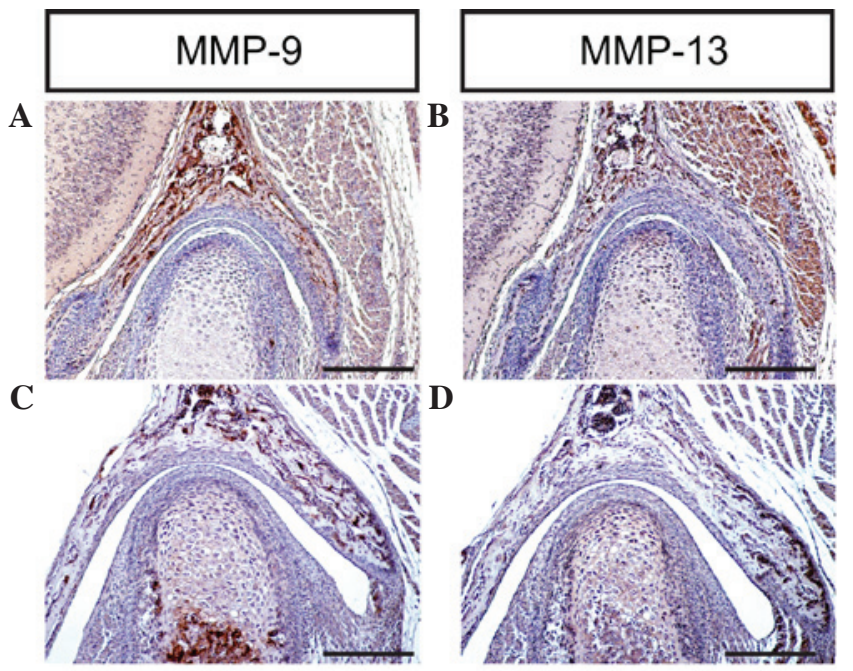

Figure 8. MMP-9 and MMP-13 expression levels were detected during temporomandibular joint (TMJ) development using immunohistochemistry at (A and B) P0 and (C and D) P7 (scale bar, $100 \mu \mathrm{m}$ ). MMP-9, matrix metalloproteinase-9, MMP-13, matrix metalloproteinase-13; P, post-natal day.

fossa undergo rapid cellular proliferation during embryonic development. The ratio of cell proliferation exhibits a gradually declining trend between days E13.5 and P0. Apoptosis, or programmed cell death, is a form of traumatic cell death that results from acute cellular injury (40). Apoptosis typically confers a number of advantages during an organism's lifecycle compared with unprogrammed cell death, including cell shrinkage, blebbing, chromatin condensation, nuclear fragmentation (41) and chromosomal DNA fragmentation. Apoptosis is responsible for the growth cartilage and endochondral ossification of condyle and glenoid fossa (Fig. 7B and C) (6,7).

Regulation of TMJ development by MMP activity. A well-coordinated remodeling of the ECM is a pre-requisite for TMJ development and homeostasis (42). The turnover of collagens and proteoglycans proceeds via various pathways, and may be dependent on endocytic processes, or involve extracellular reactions exclusively (43). ECM degradation requires the action of secreted or membrane MMPs, which are zinc-dependent endopeptidases capable of degrading ECM components, including collagens and proteoglycans (44). MMP-9 degrades denatured collagen II, which is initially cleaved by activated MMP-1, and additionally degrades noncartilage collagens such as collagen IV and collagen V (45). Notably, MMP-9 is able to degrade the core protein aggrecan $(46,47)$. MMP-13 exhibits a substrate preference for degrading fibrillar collagens, including collagen II; however, MMP-13 is also able to degrade aggrecan, a hydrodynamic molecule in the cartilage $(48,49)$. The results of the present study indicate that MMP-9 and MMP-13 are expressed in the TMJ at different stages, which is consistent with previous studies $(2,6,7,9)$, implying that MMPs are key regulators of cartilage growth and endochondral ossification during TMJ formation and development in mice (Figs. 7D and 8A-D).

In the present study, the developmental processes of the mouse TMJ were systemically investigated using HE, Safranin 
O-Fast green and Azon red/Anilin blue staining methods, and a large number of transcription factors and growth factors have been implicated in the regulation of TMJ development, such as Sox9, Runx2, Ihh, TGF- $\beta 2$ and EGF $(1-3,50)$. Using in situ hybridization, immunohistochemistry and in situ zymography methods, the expression levels of SOX-9, RUNX2, IHH, collagen I, collagen II, aggrecan, MMP-9 and MMP-13 were evaluated during the process of TMJ formation and development. In addition, the cellular proliferation and apoptosis activity during TMJ formation and development in mice were analyzed using BrdU and TUNEL assays, respectively.

Generally, the development of the TMJ in mammals has not been extensively studied. A number of factors in TMJ development remain unclear, including the profile of the signaling molecules that mediate the interactions of TMJ tissue during the developmental process, and the effects of the glenoid fossa on articular disc formation. Future studies are required, which may employ transgenic mice models in addition to experimental embryology, molecular biology and cell biology approaches, to elucidate the cellular and molecular mechanisms of TMJ development.

\section{Acknowledgements}

The present study was supported by grants from the National Natural Science Foundation of China (no. 81202645 and 81230087), the Program for New Century Excellent Talents in Fujian Province University (no. JA14150), and Fujian University of Traditional Chinese Medicine Tube Project (no. X2015021).

\section{References}

1. Purcell P, Joo BW, Hu JK, Tran PV, Calicchio ML, O'Connell DJ, Maas RL and Tabin CJ: Temporomandibular joint formation requires two distinct hedgehog-dependent steps. Proc Natl Acad Sci USA 106: 18297-18302, 2009

2. Li X, Liu H, Gu S, Liu C, Sun C, Zheng Y and Chen Y: Replacing Shox 2 with human SHOX leads to congenital disc degeneration of the temporomandibular joint in mice. Cell Tissue Res 355 345-354, 2014

3. Owtad P, Park JH, Shen G, Potres Z and Darendeliler MA: The biology of TMJ growth modification: A review. J Dent Res 92: 315-321, 2013.

4. Bravetti P, Membre H, El Haddioui A, Gérard H, Fyard JP, Mahler P and Gaudy JF: Histological study of the human temporo-mandibular joint and its surrounding muscles. Surg Radiol Anat 26: 371-378, 2004.

5. Soydan SS, Deniz K, Uckan S, Unal AD and Tutuncu NB: Is the incidence of temporomandibular disorder increased in polycystic ovary syndrome? Br J Oral Maxillofac Surg 52: 822-826, 2014.

6. Li X, Liang W, Ye H, Weng X, Liu F, Lin P and Liu X: Overexpression of Indian hedgehog partially rescues short stature homeobox 2-overexpression-associated congenital dysplasia of the temporomandibular joint in mice. Mol Med Rep 12: 4157-4164, 2015.

7. Li X, Liang W, Ye H, Weng X, Liu F and Liu X: Overexpression of Shox 2 leads to congenital dysplasia of the temporomandibular joint in mice. Int J Mol Sci 15: 13135-13150, 2014.

8. Wang Y, Liu C, Rohr J, Liu H, He F, Yu J, Sun C, Li L, Gu S and Chen Y: Tissue interaction is required for glenoid fossa development during temporomandibular joint formation. Dev Dyn 240: 2466-2473, 2011

9. Gu S, Wei N, Yu L, Fei J and Chen Y: Shox2-deficiency leads to dysplasia and ankylosis of the temporomandibular joint in mice. Mech Dev 125: 729-742, 2008.

10. Mérida Velasco JR, Rodríguez Vázquez JF, De la Cuadra Blanco C, Campos López R, Sánchez M and Mérida Velasco JA: Development of the mandibular condylar cartilage in human specimens of 10-15 weeks' gestation. J Anat 214: 56-64, 2009.
11. Yokohama-Tamaki T, Maeda T, Tanaka TS and Shibata S: Functional analysis of CTRP3/cartducin in Meckel's cartilage and developing condylar cartilage in the fetal mouse mandible. J Anat 218: 517-533, 2011

12. Wu Y, Gong Z, Li J, Meng Q, Fang W and Long X: The pilot study of fibrin with temporomandibular joint derived synovial stem cells in repairing TMJ disc perforation. Biomed Res Int 2014: 454021, 2014.

13. Gu S, Wu W, Liu C, Yang L, Sun C, Ye W, Li X, Chen J, Long F and Chen Y: BMPRIA mediated signaling is essential for temporomandibular joint development in mice. PLoS One 9: e101000, 2014.

14. Vinkka-Puhakka H and Thesleff I: Initiation of secondary cartilage in the mandible of the Syrian hamster in the absence of muscle function. Arch Oral Biol 38: 49-54, 1993.

15. Kenzaki K, Tsuchikawa K and Kuwahara T: An immunohistochemical study on the localization of type II collagen in the developing mouse mandibular condyle. Okajimas Folia Anat Jpn 88: 49-55, 2011.

16. Loughner B, Miller J, Broumand V and Cooper B: The development of strains, forces and nociceptor activity in retrodiscal tissues of the temporomandibular joint of male and female goats.

17. Owtad P, Potres Z, Shen G, Petocz P and Darendeliler MA: A histochemical study on condylar cartilage and glenoid fossa during mandibular advancement. Angle Orthod 81: 270-276, 2011.

18. Liu C, Kaneko S and Soma K: Glenoid fossa responses to mandibular lateral shift in growing rats. Angle Orthod 77: 660-667, 2007

19. Yamaki Y, Tsuchikawa K, Nagasawa T and Hiroyasu K: Embryological study of the development of the rat temporomandibular joint: Highlighting the development of the glenoid fossa. Odontology 93: 30-34, 2005.

20. Li Q, Zhang M, Chen YJ, Zhou Q, Wang YJ and Liu J: Psychological stress alters microstructure of the mandibular condyle in rats. Physiol Behav 110-111: 129-139, 2013.

21. Ricks ML, Farrell JT, Falk DJ, Holt DW, Rees M, Carr J, Williams T, Nichols BA, Bridgewater LC, Reynolds PR, et al: Osteoarthritis in temporomandibular joint of Col2al mutant mice. Arch Oral Biol 58: 1092-1099, 2013.

22. Willard VP, Arzi B and Athanasiou KA: The attachments of the temporomandibular joint disc: A biochemical and histological investigation. Arch Oral Biol 57: 599-606, 2012.

23. Gu Z, Feng J, Shibata T, Hu J and Zhang Z: Type II collagen and aggrecan mRNA expression by in situ hybridization in rabbit temporomandibular joint posterior attachment following disc displacement. Arch Oral Biol 48: 55-62, 2003.

24. Garnero P, Rousseau JC and Delmas PD: Molecular basis and clinical use of biochemical markers of bone, cartilage, and synovium in joint diseases. Arthritis Rheum 43: 953-968, 2000.

25. Zuber M, Zia F, Zia KM, Tabasum S, Salman M and Sultan N: Collagen based polyurethanes-A review of recent advances and perspective. Int J Biol Macromol 80: 366-374, 2015.

26. Natiella JR, Burch L, Fries KM, Upton LG and Edsberg LE: Analysis of the collagen I and fibronectin of temporomandibular joint synovial fluid and discs. J Oral Maxillofac Surg 67: 105-113, 2009.

27. Huang Q, Opstelten D, Samman N and Tideman H:Experimentally induced unilateral tooth loss: Expression of type II collagen in temporomandibular joint cartilage. J Oral Maxillofac Surg 61: 1054-1060, 2003

28. Aspberg A: The different roles of aggrecan interaction domains. J Histochem Cytochem 60: 987-996, 2012.

29. Gu Z, Jin X, Feng J, Shibata T, Hu J, Zhan J and Hu Y: Type II collagen and aggrecan mRNA expressions in rabbit condyle following disc displacement. J Oral Rehabil 32: 254-259, 2005.

30. Mori-Akiyama Y, Akiyama H, Rowitch DH and de Crombrugghe B: Sox 9 is required for determination of the chondrogenic cell lineage in the cranial neural crest. Proc Natl Acad Sci USA 100: 9360-9365, 2003.

31. Shibata S, Suda N, Suzuki S, Fukuoka H and Yamashita Y: An in situ hybridization study of Runx2, Osterix, and Sox9 at the onset of condylar cartilage formation in fetal mouse mandible. J Anat 208: 169-177, 2006.

32. Ochiai T, Shibukawa Y, Nagayama M, Mundy C, Yasuda T, Okabe T, Shimono K, Kanyama M, Hasegawa H, Maeda Y, et al: Indian hedgehog roles in post-natal TMJ development and organization. J Dent Res 89: 349-354, 2010.

33. Wu MJ, Gu ZY and Sun W: Effects of hydrostatic pressure on cytoskeleton and BMP-2, TGF-beta, SOX-9 production in rat temporomandibular synovial fibroblasts. Osteoarthritis Cartilage 16: 41-47, 2008. 
34. Jing J, Hinton RJ, Jing Y, Liu Y, Zhou X and Feng JQ: Osterix couples chondrogenesis and osteogenesis in post-natal condylar growth. J Dent Res 93: 1014-1021, 2014.

35. Ishizuka Y, Shibukawa Y, Nagayama M, Decker R, Kinumatsu T, Saito A, Pacifici M and Koyama E: TMJ degeneration in SAMP8 mice is accompanied by deranged Ihh signaling. J Dent Res 93: 281-287, 2014.

36. Shibukawa Y, Young B, Wu C, Yamada S, Long F, Pacifici M and Koyama E: Temporomandibular joint formation and condyle growth require Indian hedgehog signaling. Dev Dyn 236 426-434, 2007.

37. Suda N, Shibata S, Yamazaki K, Kuroda T, Senior PV, Beck F and Hammond VE: Parathyroid hormone-related protein regulates proliferation of condylar hypertrophic chondrocytes. J Bone Miner Res 14: 1838-1847, 1999.

38. Hume WJ and Thompson J: Double labelling of cells with tritiated thymidine and bromodeoxyuridine reveals a circadian rhythm-dependent variation in duration of DNA synthesis and $\mathrm{S}$ phase flux rates in rodent oral epithelium. Cell Tissue Kinet 23: 313-323, 1990.

39. Herring SW, Decker JD, Liu ZJ and Ma T: Temporomandibular joint in miniature pigs: Anatomy, cell replication, and relation to loading. Anat Rec 266: 152-166, 2002.

40. Matsuda S, Mishima K, Yoshimura Y, Hatta T and Otani $\mathrm{H}$ Apoptosis in the development of the temporomandibular joint. Anat Embryol (Berl) 196: 383-391, 1997.

41. Sato I, Uneno R, Miwa Y and Sunohara M: Distribution of tenascin- $\mathrm{C}$ and tenascin-X, apoptotic and proliferating cells in postnatal soft-diet rat temporomandibular joint (TMJ). Ann Anat 188: 127-136, 2006
42. Wattanachai T, Yonemitsu I, Kaneko S and Soma K: Functional lateral shift of the mandible effects on the expression of ECM in rat temporomandibular cartilage. Angle Orthod 79: 652-659, 2009.

43. Gao Y, Liu S, Huang J, Guo W, Chen J, Zhang L, Zhao B, Peng J, Wang A, Wang Y, Xu W, Lu S, Yuan M and Guo Q: The ECM-cell interaction of cartilage extracellular matrix on chondrocytes. Biomed Res Int 2014: 648459, 2014.

44. Okada Y: Matrix-degrading metalloproteinases and their roles in joint destruction. Mod Rheumatol 10: 121-128, 2000

45. Almeida LE, Caporal K, Ambros V, Azevedo M, Noronha L, Leonardi R and Trevilatto PC: Immunohistochemical expression of matrix metalloprotease-2 and matrix metalloprotease- 9 in the disks of patients with temporomandibular joint dysfunction. J Oral Pathol Med 44: 75-79,2015.

46. Malemud CJ: Matrix metalloproteinases: Role in skeletal development and growth plate disorders. Front Biosci 11: 1702-1715, 2006.

47. Burrage PS, Mix KS and Brinckerhoff CE: Matrix metalloproteinases: Role in arthritis. Front Biosci 11: 529-543, 2006

48. Stickens D, Behonick DJ, Ortega N, Heyer B, Hartenstein B, Yu Y, Fosang AJ, Schorpp-Kistner M, Angel P and Werb Z: Altered endochondral bone development in matrix metalloproteinase 13-deficient mice. Development 131: 5883-5895, 2004.

49. Inada M, Wang Y,Byrne MH, Rahman MU,Miyaura C,López-Otín $\mathrm{C}$ and Krane SM: Critical roles for collagenase-3 (Mmp13) in development of growth plate cartilage and in endochondral ossification. Proc Natl Acad Sci USA 101: 17192-17197, 2004.

50. Oka K, Oka S, Sasaki T, Ito Y, Bringas P Jr, Nonaka K and Chai Y: The role of TGF-beta signaling in regulating chondrogenesis and osteogenesis during mandibular development. Dev Biol 303: 391-404, 2007. 